\title{
A Brief Survey of Telerobotic Time Delay Mitigation
}

\author{
Parinaz Farajiparvar ${ }^{\dagger}$, Hao Ying and Abhilash Pandya ${ }^{*+}$ \\ Electrical and Computer Engineering, Wayne State University, Detroit, MI, United States
}

\section{OPEN ACCESS}

Edited by:

Emily Charlotte Collins,

The University of Manchester,

United Kingdom

Reviewed by:

Joaquin Carrasco,

The University of Manchester,

United Kingdom

Bin Fang,

Tsinghua University, China

*Correspondence:

Abhilash Pandya

apandya@wayne.edu

†These authors have contributed equally to this work

Specialty section: This article was submitted to Human-Robot Interaction, a section of the journal

Frontiers in Robotics and Al

Received: 01 July 2020 Accepted: 19 November 2020 Published: 15 December 2020

Citation:

Farajiparvar $P$, Ying $H$ and Pandya $A$ (2020) A Brief Survey of Telerobotic

Time Delay Mitigation.

Front. Robot. Al 7:578805

doi: 10.3389/frobt.2020.578805
There is a substantial number of telerobotics and teleoperation applications ranging from space operations, ground/aerial robotics, drive-by-wire systems to medical interventions. Major obstacles for such applications include latency, channel corruptions, and bandwidth which limit teleoperation efficacy. This survey reviews the time delay problem in teleoperation systems. We briefly review different solutions from early approaches which consist of control-theory-based models and user interface designs and focus on newer approaches developed since 2014. Future solutions to the time delay problem will likely be hybrid solutions which include modeling of user intent, prediction of robot movements, and time delay prediction all potentially using time series prediction methods. Hence, we examine methods that are primarily based on time series prediction. Recent prediction approaches take advantage of advances in nonlinear statistical models as well as machine learning and neural network techniques. We review Recurrent Neural Networks, Long Short-Term Memory, Sequence to Sequence, and Generative Adversarial Network models and examine each of these approaches for addressing time delay. As time delay is still an unsolved problem, we suggest some possible future research directions from information-theory-based modeling, which may lead to promising new approaches to advancing the field.

Keywords: teleoperation, Robotics, telesurgery, time series prediction, machine learning, recurrent neural network, Long Short-Term Memory, Sequence to sequence model

\section{INTRODUCTION}

Teleoperation or telerobotics is a broad area in robotics with a long and rich history which has been a major area of interest over the last decade with numerous applications. This form of robotics has a user at a local (master) location controlling a robot at a remote site (slave) with feedback (usually video) from that remote location. Goertz and Thompson (1954) started managing radioactive material with the help of mechanically built teleoperators and pioneered modern teleoperation in the 1950s. Interest in teleoperation has recently surged with critical applications in many domains, especially medicine (Sanchez et al., 2012; Livatino et al., 2014). The recent Covid19 pandemic has heightened the need for remote operations for both medical interventions and other logistics (Yang et al., 2020).

Examples of useful recent applications of teleoperation range from space operations, military (Chen, 2010), underwater exploration (Saltaren et al., 2007), mining, nuclear/toxic material handling, military, and robotic-assisted medical interventions (Madder et al., 2019). Figure 1 shows three applications from our laboratory, which includes three remote users of medical, space, and ground robots. A recent paper in medical robotics (Madder et al., 2019) studied the effect of time delay on a robotic coronary telestenting system. They use a robotic system at distances over 100 
A

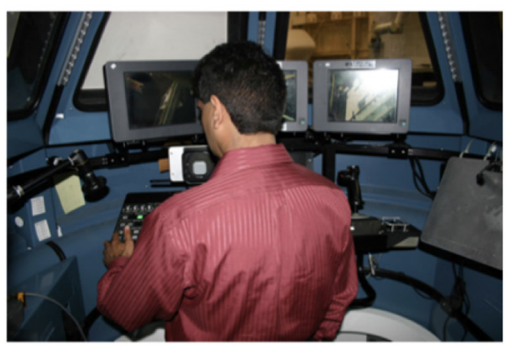

C

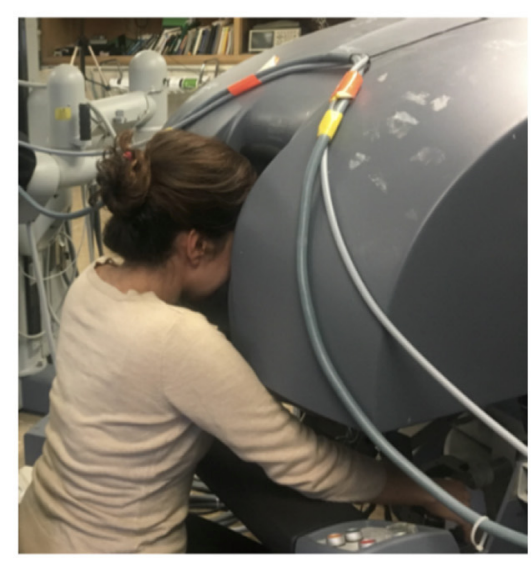

E

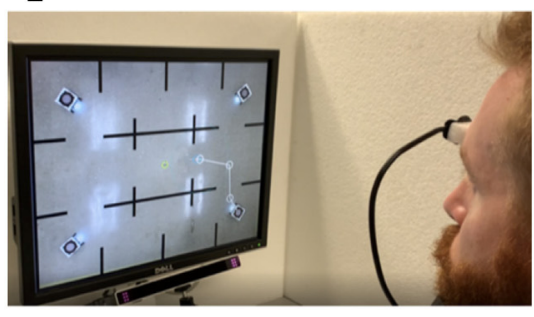

B

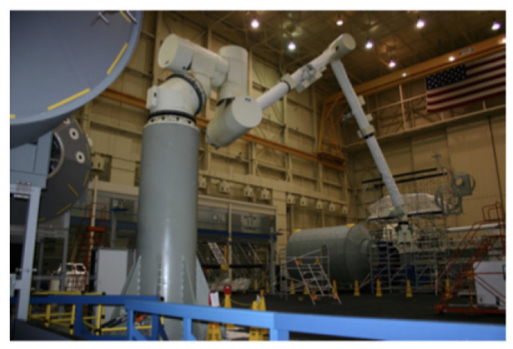

D

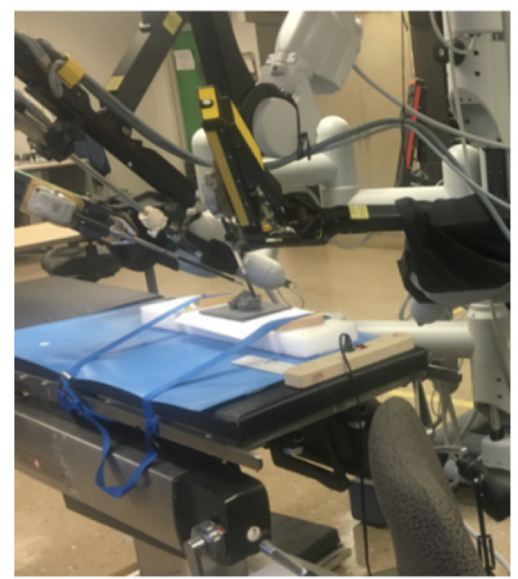

$\mathbf{F}$

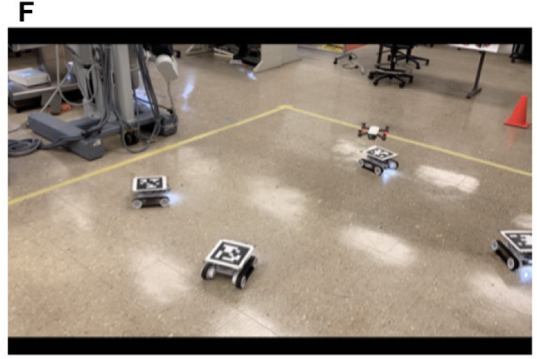

FIGURE 1 | Different applications of teleoperation. (A) Operator of a space robot arm, (B) the space robot arm (Chintamani et al., 2009). (C) Telesurgery with a research da Vinci surgical system (Eslamian et al., 2020), surgeon side. (D) Remote patient side, (E) ground robot control with aerial view (Lucas et al., 2012) operator controls ground robots using eye-tracking and (F) the ground robots (Lucas et al., 2012).

miles and simulate network latency from 0 to $1,000 \mathrm{~ms}$. They show that $400 \mathrm{~ms}$ latency is acceptable (they are able to perform

Abbreviations: AIC, akaike information criterion; AR, auto-regressive; ARIMA, auto-regressive integrated moving average; ARMA, auto-regressive moving average; convLSTM, convolutional LSTM; GAN, generative adversarial networks; GRU, gated recurrent unit; HSMM, hidden semi-Markov model; HWES, holt winter's exponential smoothing; LQG, linear quadratic gaussian; LSTM, long short-term memory networks; LSTM-VAE, LSTM and variational autoencoder; MA, moving average; MIMO, multiple-input and multiple-output; $\mathrm{NN}$, neural network; RNN, recurrent neural networks; SARIMA, seasonal autoregressive integrated moving-average; SARIMAX, seasonal autoregressive integrated moving-average with exogenous; SES, simple exponential smoothing; Seq2Seq, sequence-to-sequence; SMLR, sparse multivariate linear regressive; VARMA, vector autoregression moving-average; VARMAX, vector autoregression moving-average with exogenous regressors. stents), delays between 100 and $250 \mathrm{~ms}$ did not make a significant difference from no delay, and more than $400 \mathrm{~ms}$ delay affected the surgeon's performance. More investigation on the effect of time delay on the surgeon's performance in the medical teleoperation systems is explored by Rosen and Hannaford (2006) and Lum et al. (2009). Also, Orosco et al. (2020) found that negative motion scaling (less remote instrument movement for a particular master controller movement) improved performance for time-delayed robotic surgery.

Several recent review papers on the topic of teleoperation with time delay have been published. Sun et al. (2014) review wave variable control methods, which are an extension of passivity theory. They highlighted the issues of wave reflection and drift as barriers to this approach. Muradore and Fiorini 
(2016) review bilateral teleoperation algorithms where haptic feedback is difficult with a time-delayed interface. They review algorithms that lead to the stability of haptic interfaces based on passivity theory. Uddin and Ryu (2016) survey predictive control approaches to mitigate time delay. They cover modelbased approaches mostly related to predictive control. Kebria et al. (2018) review internet-based teleoperation systems. They deal with issues of delay, packet loss, jitter, and blackout seen in internet-based approaches and cover traditional and adaptivebased control approaches, neural networks, and fuzzy-based approaches where they model the uncertainty by modeling time delay.

In this paper, we review the trends and strategies used to solve the time delay issues related to teleoperation. First, we will briefly review some of the early approaches and examine predictive and statistical approaches. Then we explore the closely related field of time series prediction using machine learning and AI techniques and how they have been or can be applied to this field. We will also provide our analysis and projection for open research questions and on future research directions that can utilize the recent explosion and progress in deep neural networks, machine learning, and information theory.

In section 2, we review the early approaches for the time delay in teleoperation, which consist of primarily control theory approaches. In section 3, we introduce the time series prediction problem and highlight the similarities between the time delay problem and the general time series problem. In this section, we review statistical models and Neural Networks (NN) based methods. Finally, in section 4, we compare and discuss the methods and conclude with some future avenues of research and new ideas to solve time delay in teleoperation.

\section{TRADITIONAL APPROACHES TO MITIGATING TIME DELAY IN TELEOPERATION}

Early research in the field deals primarily with creating appropriate user interfaces for teleoperation (Sheridan, 1992) and issues related to the communication channels between the remote and local sites. Due to distance, low speed, poor quality of the communication channels, and time delay, data corruption often occurs in teleoperation systems. The main issue that is researched in the literature is the time delay of communication channels. This is a fundamental problem related to signal transmission's physical limits and not necessarily on the hardware. Data transmission delay in teleoperation can be between less than a few milliseconds to many minutes based on the distance between the master and slave locations and the communication medium. Excessive delays can make teleoperation very difficult to perform. Research techniques to stabilize and mitigate time delay issues have been a significant area of interest in teleoperation.

\subsection{Defining a Model for Time Delay}

Ferrell and Sheridan's experiments (Ferrell, 1965) determined the impact of time delay on human operator performance in teleoperated manipulators. They used the servo-driven manipulator with two parallel slave fingers controlled by a human operator (master). In this experiment, the time delay is added to command signals from the human operator before it is received by the slave. Their research showed that operators respond to the delay with a move and wait strategy. That means that the operator moves the joystick and waits for feedback before responding again to the remote robot. Then the user starts a corrective step and waits again to recognize the remote system's delayed reaction and repeats the cycle until the operation is complete. Based on Hokayem and Spong (2006), the completion time of an assignment is defined as follows:

$$
t(I)=t_{r}+\sum_{i=1}^{N(I)}\left(t_{m i}+t_{w i}\right)+\left(t_{r}+t_{d}\right) N(I)+t_{g}+t_{d}
$$

where $I$ is the measure of difficulty, $N(I)$ is the number of the movements, $t_{r}$ is the human's reaction time, $t_{m i}$ is the movement duration, $t_{w i}$ is the waiting time after each move, $t_{g}$ is the grasping time and $t_{d}$ is the delay time introduced into the communication channel. The completion time for a particular assignment depends linearly on the delay factor in the control loop; therefore, the longer the delay, the greater the completion time. Ferrell and Sheridan's experiments (Ferrell, 1965) conclude that the move and wait strategy works; however, it takes a longer time and has smoothness implications.

\subsection{Supervisory Control Methods}

Supervisory control with a direct connection between the master and remote sides was introduced by Ferrell and Sheridan (1967), to address the time delay problem. In supervisory control, based on the difficulty of the task and the order of the autonomy of the process, the control could be either of symbolic or analog nature. In the symbolic approach, they introduce small autonomous sub-tasks, which are high-level commands used for the local controller. Whitney (1969) introduced the supervisory methodology from an optimization perspective by developing a discrete-state-space and applying search strategies to accomplish the ideal performance of the task.

With the advancement in microprocessor design and programming, yet another solution was introduced for the time delay in teleoperation. In this approach, a processor (at the remote site) was able to do simple tasks like close the gripper or move it from point $\mathrm{A}$ to point B. Other complex tasks could be performed by chaining the simple commands together. In this way, only high-level task commands need to be sent. Modular software for simple or repetitive tasks was introduced in this area and improved the performance and minimized communication time by using inter-processor communication and control mode selection (Fong et al., 1986). Task-Oriented Supervision Command System (Madni et al., 1983), and the language-aided robotic teleoperation system in Sato and Hirai (1987) provide a method to do simple tasks. In addition, visual models helped control process by adding graphics models of the motion of the robot (Stark et al., 1987; Hirzinger et al., 1989; Bejczy and Kim, 1990). Bejczy et al. (1990) used the phantom robot models to predict real robot motion. Buzan and Sheridan 
(1989), applied a predictive operator aid to handle the time delay in telemanipulator systems.

\subsection{Predictive Control-Based Approaches for Time Delay}

Time delay is a challenge when designing stable controllers. Control solutions for time-delayed teleoperation systems open up new questions for the interpretation of the fundamental principles of control systems and render it necessary to reevaluate these ideas. In many instances, traditional techniques fail to stabilize systems, especially when the time delay varies or blackout occurs. The impact of time delay on the stability of a remotely controlled system was studied by Varkonyi et al. (2014). The authors consider a remotely controlled system where keeping the system stable with time delay was a challenge. Varkonyi et al. (2014), they showed that traditional control system methods are not successful when there is a variable time delay or communication blackout.

A good review, Uddin and Ryu (2016), provides a comprehensive comparison and analysis of different approaches for predictive control. This paper covers mostly modelbased approaches for teleoperation. A multi-model predictive controller was proposed by Sirouspour and Shahdi (2006). They propose a discrete linear quadratic Gaussian (LQG) controller for teleoperation with time delay in communication (Sirouspour, 2005). In this case, the sampling rate was restricted as the delay increases in order to decrease the computational load and prevent potential numerical issues. This method has limits as the closed-loop reaction and teleoperation stability can be adversely affected by a small sampling rate.

Sirouspour and Shahdi (2006) propose a novel approach to the reduction and output-feedback control of MIMO (multiple-input and multiple-output) systems with nonidentical delay and they show that this system acquires the detectability and stabilizability properties of the original system. Their second achievement in that paper was that they formulate teleoperation under delay as a multi-model continuous-time LQG synthesis problem using the proposed output-feedback control approach.

\subsection{Passivity-Based Methods}

It is possible to model the delay problem mathematically as a "passivity-based teleoperation." Desoer and Vidyasagar (1975) introduce passivity-based teleoperation in bilateral teleoperation to ensure stability and performance with packet loss and time delay. There are different passivity-based approaches to model the master-slave teleoperator system, such as 2-port networks (Buzan and Sheridan, 1989), impedance matrix (Raju et al., 1989), hybrid matrix (Hannaford, 1989), scattering approach (Anderson and Spong, 1992), constant time delay (Anderson and Spong, 1992), scaling (Colgate, 1991), wave variables (Niemeyer and Slotine, 1991a,b; Benedetti et al., 2001; Ganjefar et al., 2002; Munir and Book, 2002), and geometric scattering (Stramigioli et al., 2002). Hokayem and Spong (2006) examined the theoretical control approaches to address the time delay problem and information loss. In passivity-based teleoperation, researchers represent the master/slave teleoperation system with linear models. Nuño et al. (2011) review several passivitybased controllers for non-linear bilateral teleoperation. Polat and Scherer (2012) presents stability analysis for uncertain bilateral teleoperation systems using the IQC framework. They formulate stability using network theory and run numerical test cases to verify their formulation. In addition, Tugal et al. (2016) investigate the stability of passive multipliers and Zames-Falb multipliers with the IQC framework for both time-invariant and time-variant time delays.

Another kind of teleoperation that was introduced is internetbased teleoperation (Goldberg et al., 1995; Kebria et al., 2018). Different approaches to deal with random time delay in message transmission over the internet have been researched. This area of real-time communication for teleoperation over the internet has been active since the 1990s. Xi and Tarn (2000) propose nontime referenced action control method to deal with random time delay, and Oboe (2003) show the success of real-time closed-loop control systems for telerobotics over the internet.

Furthermore, predictive techniques are introduced to model and mitigate the random time delay with fuzzy adaptive control methods by Lu et al. (2017) and Mirfakhrai and Payandeh (2002) where they used an autoregressive model. Ye et al. (2002) and Shen et al. (2019) used nonlinear time series analysis to understand the delay behavior and estimate time delay. The application of neural networks for creating a robust teleoperation system with time-varying communication delay was studied by $\mathrm{Li}$ and $\mathrm{Su}$ (2013). These papers opened up a new area of research with time series analysis/prediction. We will explain time series prediction and the different avenues of research in the next section.

In summary, control-based methods to mitigate time delay for teleoperation systems can be divided into two approaches. In the first approach, guaranteeing stability is used to deal with variable time delay. In addition, Passivity-based control methods are applied for modeling master-slave teleoperator systems. A second approach is a predictive approach, which consists of model-based approaches like the LQG controller (Sirouspour, 2005; Sirouspour and Shahdi, 2006). This approach has shown improvement in performance in terms of stability and transparency. Another example of this approach is when a processor performs complex tasks by chaining simple commands together (Hirzinger et al., 1989). That method could take advantage of a time series prediction to further mitigate time delay.

\section{TIME SERIES PREDICTION METHODS FOR MITIGATING TIME DELAY IN TELEOPERATION}

An avenue of research in the time delay problem in teleoperation is using time-series predictions, where time-series predictions are used to compensate for the time delay. In time series prediction, the goal is to predict future values based on past observations which consist of intrinsic patterns. To determine a model that expresses the pattern of time series, we need a method to describe 


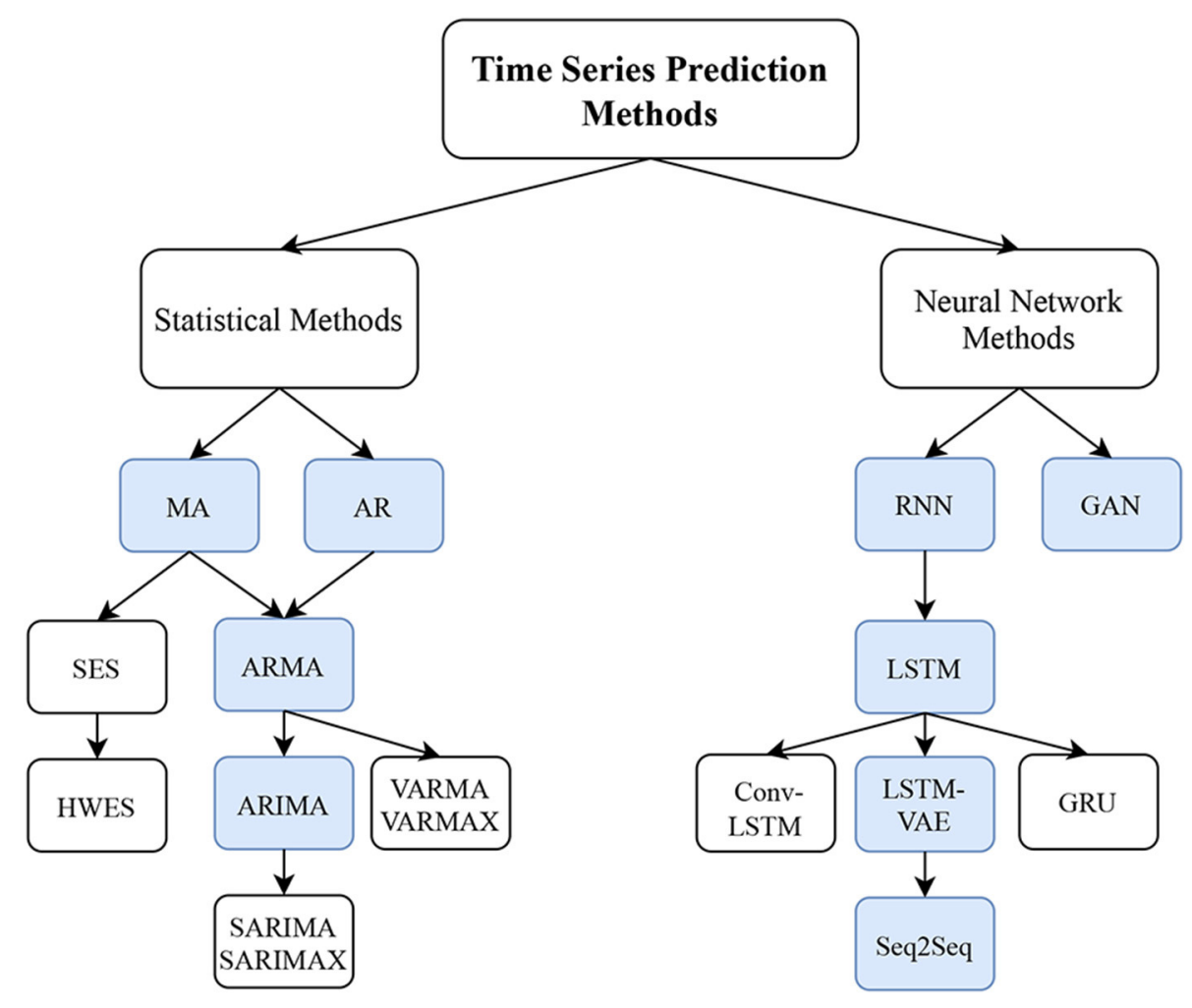

FIGURE 2 | Hierarchical structure of time series prediction techniques in both statistical and machine learning approaches. For statistical methods, our main focus is on the blue boxes (i.e., AR, MA, ARMA, and ARIMA) as they are the more fundamental methods. The remaining methods are their extensions. For Neural Network Methods, we focus on the RNN, LSTM, Seq2Seq, and GAN methods, which are in blue boxes.

the important features of the time series pattern and explain how the past affects the future or how two-time series can "interact."

In general, time series have three major common patterns:

1. Trend: The specific direction of the time series can be a longterm increase or decrease in the data (Parmezan et al., 2019).

2. Seasonality: The repetitive patterns at predictable intervals.

3. White Noise: The unpredictable fluctuation with no seasonality or trend.

In this paper, we divide time series prediction methods into two main methods: (as shown in Figure 2): Statistical approaches, Neural Network approaches. We cover these two methods as recent approaches for time series prediction.

\subsection{Examples of Time Series Prediction in Delay Mitigation}

Several early approaches for delay mitigation used time series prediction. Mirfakhrai and Payandeh (2002) employed an autoregressive model to forecast future values of time delay. The predictions were used with a look-up table to tune the system by adjusting the gain and decreasing the mismatch between forces and velocities at the master and slave sides. In their study, the slave side is assumed to be precisely similar to the master side; also, the slaves' delay and master communication are equal, and there is no scaling between the master and slave. Ye et al.
(2002) studied the delay for round trip time (RTT) in internetbased communication. They use a linear correlation of RTT by computing the autocorrelation and power spectrum. In this paper, the authors used the maximum entropy principle (MEP) which is a linear algorithm to predict one step ahead of the RTT value.

Recently, there have been several papers that use timeseries predictions for teleoperation time delay mitigation. For instance, Chen et al. (2020) utilizes a statistical approach using a multivariate linear regression model to forecast time delay for space teleoperation. When time delay is known in advance, control of remote systems is improved in comparison with the previous approaches that included sparse multivariate linear regression (SMLR) (Chen et al., 2019), autoregressive (AR), neural network (NN), and cubic polynomial model-based (CPMB) approaches. Su et al. (2020b) apply deep convolution neural networks to identify a robot tool's dynamics for bilateral teleoperation. Belhaj and Tagina (2009) apply RNNs to model and predict internet end-to-end time delay. Su et al. (2020a) propose an improved RNN to predict the trajectory of manipulators. Aburime et al. (2019) applied recursive least squares filtering to identify the delay and target waypoints. They were able to show that an aerial vehicle can estimate a waypoint based on an appropriate filter selection while it also monitors the user commands. Zheng et al. (2019) also use a similar approach for a 
ground vehicle heading prediction. They use a blended approach with a Taylor series expansion with estimated noise to model the heading for remote-controlled vehicles. They use the delayed signals to predict the heading of the vehicle such that in the event of a delay, the vehicle does not go off course. Arita and Suzuki (2019) employ an exponential statistical prediction model of human gaze points to assist in the maneuvering of teleoperated robots. They use a first-order gaze movement model with a time delay to predict specific target points and use these as points of movement during the time delay. Jung et al. (2019) utilize a predictive display using a kinematic model of the human head and neck along with camera parameters. This is an interesting approach where they use the relationship between camera orientation and the human controllers' positional changes to predict future images based on current images using a simple linear model of motion.

\subsection{Statistical Models for Time Series Prediction}

In this subsection, we discuss the statistical methods for time series prediction. We also explain the use of statistical models to try and predict the near-term future to help mitigate time delay. This approach uses predictions based on statistical methods to model incoming data with the aim of predicting the next steps and replacing the data which is not received because of time delay.

Statistical models summarize the data with equations that represent a relation between input and output (Wasserman, 2013). A predictive statistical model defines a process on data to predict new or future observations (Shmueli, 2010) based on the observed data. The evolution of statistical techniques started with a simple technique of linear auto-regression (AR) (Mills and Mills, 1991). This technique uses a model that predicts future steps based on a linear model of the past data. Similarly, some techniques use a moving average (MA) as a predictor of future events (Box and Pierce, 1970). This technique also uses a stochastic component that models noise in the system. Both the $\mathrm{AR}$ and MA techniques were combined to create a system that was named ARMA. In this method, predictions were based on both a linear regression as well as a moving average. The next evolution added a nonlinear component (integration) to the formulation and was named ARIMA. The addition of the integral term allowed better prediction of non-stationary signals (Mondal et al., 2014). Simple Exponential Smoothing (SES) is an extension of the MA method. It uses an exponential window function, which exponentially weights previous observations in the time series. It differs from MA which treats all previous steps equally. Holt Winter's Exponential Smoothing (HWES) is a triple exponential smoothing method that predicts the next time steps based on three factors-the weighted prior time step, the trend and the seasonality (Kalekar, 2004). Vector Autoregression Moving-Average (VARMA) is the generalization of the ARMA model to forecast a multivariate time series. Vector Autoregression Moving-Average with Exogenous Regressors (VARMAX) is the extension of the VARMA model along with the exogenous variables or covariates as the parallel independent input sequences (Poskitt, 2016). Seasonal ARIMA or Seasonal Autoregressive Integrated Moving-Average (SARIMA) is an extended version of ARIMA with the ability to capture the seasonality of the time series (Hyndman and Athanasopoulos, 2018) and Seasonal Autoregressive Integrated Moving-Average with Exogenous Regressors (SARIMAX) is a method with exogenous data to improve the prediction result. In the next section, we will cover the details of these four methods: AR, MA, ARMA, ARIMA. All the other methods are extensions of these four methods and shown in Figure 2 as white boxes. The details of these extensions are not covered extensively as they are closely related to the core methods.

\subsubsection{AR Model}

Auto-regressive (AR) models describe the linear dependency of the previous values to the predicted values. The basic components of an AR model include linear coefficients for prediction, a model of error (noise) in the system, and a method to determine the extent of the past data needed for prediction based on an autocorrelation function. The first-order Auto-regressive model $\operatorname{AR}(1)$ is the linear model between the value of time step at time $x_{t}$ and previous time step $x_{t-1}$ which includes coefficients $\left(\phi_{0}, \phi_{1}\right)$ with assumption $\left|\phi_{i}\right|<1$, where $i=1,2$ and noise at time $t, \omega_{t}$ is defined as:

$$
x_{t}=\phi_{0}+\phi_{1} x_{t-1}+\omega_{t}
$$

where $\omega_{t} \sim N\left(0, \sigma_{\omega}^{2}\right)$ (i.e., Gaussian distribution with zero mean and $\sigma_{\omega}^{2}$ as a variance) is the present error and $\phi_{1}$ is the slope of $\operatorname{AR}(1)$.

In general an $A R(p)$ model (where $p$ is the order of the model which can be selected as a design parameter) is given by the following equation:

$$
x_{t}=\phi_{0}+\phi_{1} x_{t-1}+\phi_{2} x_{t-2}+\cdots+\phi_{p} x_{t-p}+\omega_{t}
$$

Mirfakhrai and Payandeh (2002) and Hu et al. (2012) use this auto-regressive model to predict the value of time delay in the future. In their model $x_{t}$ is the signal that is modeled and $\omega_{t}$ is the white noise with auto-correlation. To determine the order of the AR model researchers use the Partial Autocorrelation Function (PACF) (Mishra and Desai, 2005). The PACF shows the autocorrelation between the variable and a lag without considering the effect of lower-order lags. For example, when the PACF tends to zero at $k$ lags, it shows the order of our AR model $p$ is equal to $k$. Once an appropriate order has been determined, a model can be formed to allow prediction.

\subsubsection{MA Model}

Another method for modeling the time series data is the moving average (MA) model. The MA model takes the average of the previous values of the time series to predict future values. The basic components of an MA model are similar to the AR model except that in this model, we want to know how much noise in the past affects the prediction. The first order of an MA model is denoted by MA(1) and is defined as:

$$
x_{t}=\mu+\omega_{t}+\theta \omega_{t-1}
$$


where $\mu$ is the mean of the time series, $\theta$ is the parameter of the model and $\omega_{t}$ is the white noise with zero mean and $\sigma_{\omega}^{2}$ as a variance.

General MA(q) models:

$$
x_{t}=\mu+\omega_{t}+\theta_{1} \omega_{t-1}+\theta_{2} \omega_{t-2}+\cdots+\theta_{q} \omega_{t-q}
$$

A property of $\mathrm{MA}(\mathrm{q})$ models in general is that there are nonzero autocorrelations for the first $q$ lags and autocorrelations $=0$ for all lags $\geq q$. Similar to AR, in the MA model, we can find the order of the model based on the Autocorrelation Function (ACF). The ACF shows the autocorrelation of a variable and a lag of itself (Moayedi and Masnadi-Shirazi, 2008). Once an appropriate order has been determined, a model can be formed to allow prediction.

\subsubsection{ARMA Model}

The AR model is a prediction based on the lags of the data. The MA model is a prediction based on the past noise of the signal. In order to create a more general model that incorporates both these features, researchers combined these techniques (Choi, 2012). The combination of AR and MA models creates an autoregressive moving average model ARMA. There are three basic steps of creating an ARMA model. The first is, model selection, the second is parameter estimation and the third is model checking (Box et al., 2015). Model selection and parameter estimation are challenging problems in time series prediction. Rojas et al. (2008) introduced a method to determine the linear model automatically. An $\operatorname{ARMA}(p, q)$ model (where $\mathrm{p}$ and $\mathrm{q}$ are model orders) can be defined based on the $\mathrm{AR}(\mathrm{p})$ and $\mathrm{MA}(\mathrm{q})$ equations as follows:

$$
x_{t}=\phi_{1} x_{t-1}+\cdots+\phi_{p} x_{t-p}+\omega_{t}+\theta_{1} \omega_{t-1}+\cdots+\theta_{q} \omega_{t-q}
$$

where the $\omega$ is the noise and the $\phi$ are the coefficients of AR and the $\theta$ are the coefficients of MA. The ARMA model can be used to predict stationary time series which have constant stochastic properties (mean, variance and correlation) with respect to time. Real-world time series data cannot be guaranteed to be stationary. Hence, a term needs to be added to make the model that can fit nonlinear non-stationary signals. Hua et al. (2013) employ this statistical method to estimate the time delay of a one-way internet connection.

\subsubsection{ARIMA Model}

By adding a differentiation term (d) to the ARMA model, researchers converted the non-stationary signal to a stationary signal. The difference term added to a non-stationary signal makes it stationary, and this is commonly referred to as an integrated signal. Hence, this technique with the integrated signal addition is called the ARIMA model and is used for nonstationary time series prediction (Lorek and Willinger, 1996).

The $\operatorname{ARIMA}(p, d, q)$ model, is the combination of Autoregression, $\mathrm{AR}(\mathrm{p})$, integration, and Moving Average, $\mathrm{MA}(\mathrm{q})$. Here, $p$ refers to the order of autoregression, $d$ is the degree of difference and $q$ is the order of the moving average terms.
In order to assess the trade-off between the goodness of fit and over-fitting, researchers have introduced a measure called the Akaike Information Criterion (AIC). AIC is used to compare the quality of statistical models. Based on Montgomery et al. (2015, Equation 2.44), AIC is used as a criterion to select the best ARIMA model parameters and is defined as:

$$
A I C=-2 L+(\log (n)+1) k
$$

where the $L$ is the likelihood function logarithm, $n$ is the number of observations, and $k$ is the number of estimated parameters. A smaller AIC points to a better/more optimized model. AIC increases if the model is overfitting. Hence, AIC analysis allows us to balance our model between adding more parameters (at the cost of over-fitting) and better fitting.

Although ARIMA is the most general statistical model for forecasting in time series, it cannot deal with all nonlinear relationships like seasonality. Seasonal ARIMA or SARIMA is an extended version of ARIMA with this ability to capture the seasonality of the time series. There are various features of time series which can be analyzed with non-parametric (Aneiros-Pérez and Vieu, 2008) or hybrid models (Faruk, 2010). In addition, some machine learning algorithms were also introduced for the time series problem. The evolution of time series prediction using machine learning techniques has had several avenues of research and development in teleoperation and robotics. Yang et al. (2018) used a hidden semi-Markov model (HSMM) and Gaussian mixture models to improve the performance of teleoperation systems. In the following section, we see how this work was extended and review some neural network models for time series prediction.

\subsection{Neural Network Methods for Time Series Prediction Problem}

In this section, we examine various neural network-based methods. Prediction of individual sequences in terms of time or time series prediction is challenging and at the same time an important area of study in machine learning (Giles et al., 2001; Anava et al., 2013, 2015; Ak et al., 2015; Fang et al., 2017, 2020). Extracting good representative pairs of input and output data is essential in machine learning algorithms. These pairs are then used to train various types of neural network architectures. There are inherent properties of certain structures that make these predictions more accurate. Time series prediction uses models known as sequential data models. These sequential data models must maintain a particular order of the data streams.

Recurrent Neural Networks (RNNs) was among the first type of neural networks method for time series prediction (Mikolov et al., 2010). These networks then evolved to Long Short-Term Memory networks (LSTM) in order to model the past dependency in a more rigorous way (Hochreiter and Schmidhuber, 1997). A further refinement in neural network architecture was made with sequence-to-sequence (Seq2Seq) modeling. This architecture used LSTM in novel ways to improve time series prediction (Sutskever et al., 2014). Other neural network methods for time series prediction are Gated Recurrent 


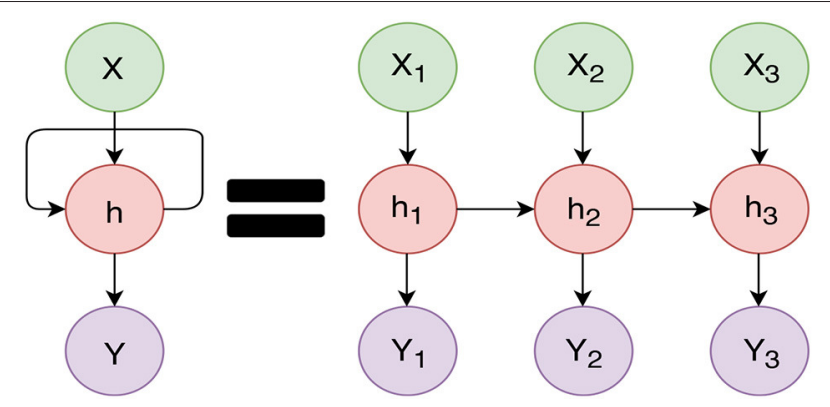

FIGURE 3 | Recurrent Neural Network with feedback (left). Unfolding of the Recurrent Neural Network (right): The $X$ values are the inputs (past values of the signal). $Y$ values are the predictions. Each successive $X$ is considered when predicting the next $Y$ value. For instance $Y_{1}$ is predicted based on $h_{1}$, but $Y_{2}$ is predicted based on both $h_{1}$ and $h_{2}$, and $X_{2}$.
Unit (GRU) (Cho et al., 2014), LSTM and Variational AutoEncoder (LSTM-VAE) (Park et al., 2018), convolutional LSTM (convLSTM) (Karim et al., 2017), and Generative Adversarial Networks (GANs) for time series prediction (Yoon et al., 2019).

In the following section, we will review the basics of RNNs, LSTM, LSTM-VAE, Seq2Seq, and GAN techniques for time series prediction.

\subsubsection{RNN Model}

RNNs are different from feed-forward (traditional) neural networks in that they have a closed feedback loop and contain an element of memory. RNNs can remember the past with the use of a loop construct. This loop allows RNNs to persist the information. We can imagine the RNN as multiple copies of the same network, as shown in Figure 3. RNNs work well when we want to look at the recent information to predict the future. Connor et al. (1994) proposed a robust learning algorithm based on filtering anomalies from the data and used this filtered data for estimating parameters and do forecasting. In Giles et al. (2001), they examine the difficulties of RNNs for forecasting nonstationary and noisy data, and they introduced a pre-processing method to overcome these problems. However, when the gap between the relevant information and the prediction is large, RNNs become very slow and, in some cases, are unable to learn the long-term dependencies (Bengio et al., 1994).

\subsubsection{LSTM Model}

RNNs have issues with long-term dependencies and vanishing gradients. In order to deal with these issues (Hochreiter and Schmidhuber, 1997) introduced the LSTM networks (Figure 4). In this model, each ordinarily hidden layer is changed by adding a memory cell. We call these kinds of networks LSTM because it has long-term memory in terms of the weights and these weights can be altered by training. In addition, it also has short term memory (implemented by gates) in terms of a temporary activation (Lipton et al., 2015). Hence, in the LSTM, we have two main parts of the network the "forget gate" and "input gate." The forget gates decide which information should be pushed away

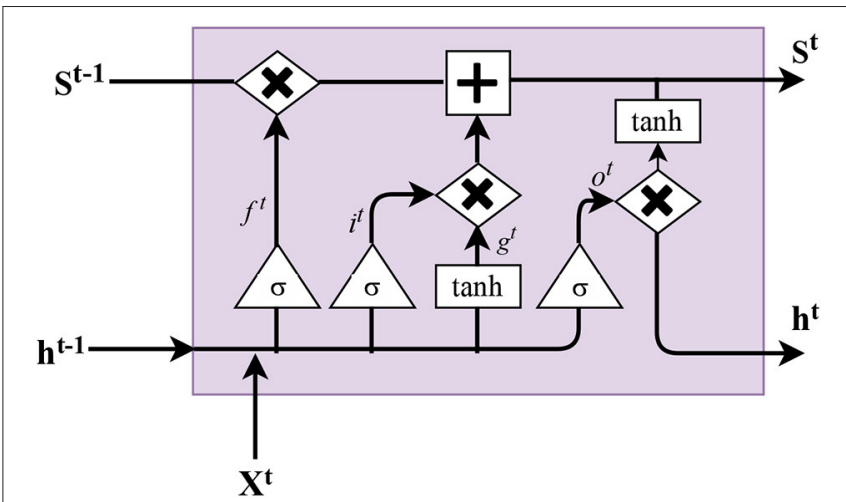

FIGURE 4 | LSTM memory cell. Inputs: current input $x^{t}$, state of previous time step $S^{t-1}$ and output of previous time step $h^{t-1}$. Outputs: updated state $S^{t}$ and current output $h^{t}$.

from the cell state, and the input gate decides which information needs to be stored in the cell state.

In each step, the forget gate considers the previous output $h^{(t-1)}$ and input $x^{t}$. The output of this forget gate is a number between 0 or 1.1 represents "keep the value," and 0 represents "forget value." The following equation represents the forget gate:

$$
f^{(t)}=\sigma\left(W_{f}\left[x^{(t)}+h^{(t-1)}\right]+b_{f}\right)
$$

where $\sigma$ is the sigmoid function, $h, W_{f}$, and $b_{f}$ are hidden layer, weight and bias of the forget gate, respectively. In order to decide what part of the information we have to keep and use in the cell state at the next time step, we need the "input gate" $i^{t}$ to specify which values we are updating. Then another gate with tanh as the activation function ( of the new candidate values to be added to the state cell $S^{t}$. As a result, this method updates the new state based on multiplying the forget gate by the previous state and multiplication of input gate $g^{(t)}$ as follow:

$$
S^{(t)}=f^{(t)} \odot S^{(t-1)}+g^{(t)} \odot i^{(t)}
$$

where the $\odot$ is element-wise multiplication. Finally, we have to determine the "output gate" $o^{t}$, which relies on the previous output $h^{(t-1)}$ and the input $x^{t}$. Then the updated output $h^{t}$ is calculated by multiplication of the "output gate" and tanh of the updated cell state as follows:

$$
h^{(t)}=o^{(t)} \odot \tanh \left(s^{(t)}\right)
$$

There are a lot of extensions of the LSTM memory cells which are used for different time-series applications like convLSTM (Kim et al., 2010; Xingjian et al., 2015) for weather forecasting and video prediction (Lotter et al., 2016). Gated Recurrent Unit (GRU) is a simple version of LSTM (Cho et al., 2014). It combines the forget and input gate of the LSTM and reduces the complexity by decreasing the number of parameters. 


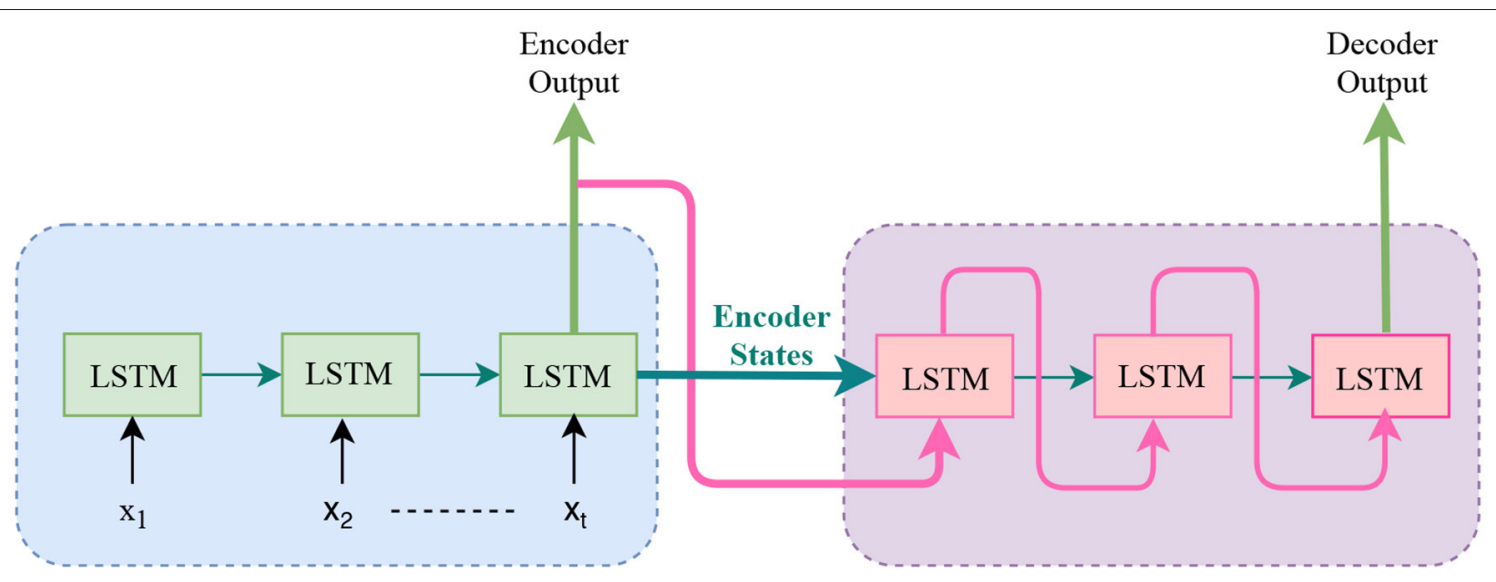

FIGURE 5 | Sequence to Sequence model: This mode uses "encoder-decoder" and maps input sequence with "encoder states" and use it as initial state of the decoder.

\subsubsection{LSTM-VAE Model}

Another framework of LSTM is the LSTM-VAE, which is a neural network based on both a variational autoencoder to compress the input and an LSTM for prediction. The encoderdecoder LSTM architecture for time series data is used by Park et al. (2018) for multi-modal anomaly detection for robot assistant feeding. Park et al. (2018) employed the LSTM unit for modeling the dependencies in the time series, as a variational autoencoder models the probability distribution of observations using variational inference (VI). LSTM has the advantage of both short and long term memory. However, in the case of multiple time series step predictions with dynamic output lengths, the system needs retraining to get new model parameters due to the requirement of different output lengths. In the next section, a new LSTM-based model called Seq2Seq is introduced. It can allow and improve dynamic multiple time series prediction.

\subsubsection{Seq2Seq Model}

A Seq2Seq model was first introduced by Sutskever et al. (2014) as a learning method for natural language processing. This translation application is like time series prediction because it has a sequential model and it also has sequential input-output pairs. In their method, they use a multi-layered LSTM to map the input sequence to a vector with a fixed dimension. Then this vector is used by another deep LSTM to decode the target sequence from that vector. The sequence to sequence model consists of two blocks of LSTMs, which are incorporated into an encoder and decoder block, as shown in Figure 5. Their main result was that on an English to French translation tasks from the WMT-14 dataset, they produced a good result compared with other methods.

Mariet and Kuznetsov (2019) provided a theoretical study for time series prediction with sequence to sequence models. They found the generalization bound of sequence to sequence models for time series prediction. Furthermore, they proposed a measurement based on the sequence data properties to determine whether the Seq2Seq model can be successful. Seq2Seq models are also used extensively for the dynamic, spatial-temporal characteristic of multivariate time series data (Zhu and Laptev, 2017; Du et al., 2018; Yang et al., 2019; Salinas et al., 2020). Another application of the sequence to sequence model is in video and image captioning (Venugopalan et al., 2015; Yang et al., 2017).

\subsubsection{GAN Model}

Generative Adversarial Network (GAN) is a kind of neural network which was introduced by Goodfellow et al. (2014) as a framework to estimate a generative model using an adversarial operation. This structure consists of two neural networks. First, a generative network produces new data based on the distribution of a training set. Second, a discriminative model evaluates the probability that a sample was drawn from it. Therefore, in time series prediction, it can generate new time series samples with the same distribution. GANs are quite new and were introduced and found suitable for unsupervised learning (Radford et al., 2015), deep reinforcement learning (Ho and Ermon, 2016) and semi-supervised learning (Donahue et al., 2016). There is an extensive exploration in time series prediction using GAN. Zhang et al. (2019) compare GAN with baseline models from ARIMA. They also compare shallow and deep LSTMs with GAN for stock market price predictions. Based on the result of this paper, the best performance is with a shallow LSTM; however, GAN has an acceptable performance. ForGAN is a model based on a conditional GAN with an LSTM/GRU layer and was proposed by Koochali et al. (2019) as a novel approach for forecasting future values. Another GAN model for time series prediction introduced by Zec et al. (2019). This model is a recurrent conditional GAN and uses LSTMs both as the generator and discriminator to express the long-term dependencies in time series. In this approach, they trained the network using the Seq2Seq method. Furthermore, Yoon et al. (2019) proposed the TimeGAN framework to capture the temporal dynamics of the time series data. They compared their novel network with Conditional and Recurrent GAN. Recently GAN-based architectures have become more popular for sequential data due to the generative property of these models. However, most papers and research in the time series prediction field still employ RNNs or LSTM. 
TABLE 1 | Comparison of the selected methods reviewed in this paper.

\begin{tabular}{|c|c|c|c|c|}
\hline Method & Linearity & Stationarity & Advantage & Disadvantage \\
\hline AR & Linear & Stationary & Applies lags and shifts of historical data; simplicity & $\begin{array}{l}\text { Unsuitable for nonlinear and non-stationary signals; } \\
\text { susceptibility to noise }\end{array}$ \\
\hline MA & Linear & Stationary & Reliable result for stationary signal & $\begin{array}{l}\text { Unsuitable for nonlinear and non-stationary signals; can } \\
\text { only predict one step in the future }\end{array}$ \\
\hline ARMA & Linear & Stationary & AR model with a MA to improve the result & $\begin{array}{l}\text { Not appropriate for long-term prediction and } \\
\text { non-stationary signals }\end{array}$ \\
\hline ARIMA & Nonlinear & Non-stationary & $\begin{array}{l}\text { Promotes ARMA by adding an integral term to handle } \\
\text { non-stationery }\end{array}$ & $\begin{array}{l}\text { Unsuitable for long-term prediction, cannot fully capture } \\
\text { the non-linearity }\end{array}$ \\
\hline RNN & Nonlinear & Non-stationary & Utilizes the saved information in the past via feedback & $\begin{array}{l}\text { Long-term dependencies; vanishing gradient and } \\
\text { exploding gradient }\end{array}$ \\
\hline LSTM & Nonlinear & Non-stationary & $\begin{array}{l}\text { Solves long-term dependencies and vanishing gradient } \\
\text { in RNN }\end{array}$ & $\begin{array}{l}\text { More prone to overfitting, longer training time and require } \\
\text { more memory to train because of more parameters }\end{array}$ \\
\hline Seq2Seq & Nonlinear & Non-stationary & $\begin{array}{l}\text { Better mapping of input and output relationships; } \\
\text { suitable for nonlinear time series }\end{array}$ & $\begin{array}{l}\text { More parameters in comparison with LSTM, slower } \\
\text { learning }\end{array}$ \\
\hline GAN & Nonlinear & Non-stationary & $\begin{array}{l}\text { Generative inheritance to learn the distribution of time } \\
\text { series, shows good results for temporal setting }\end{array}$ & $\begin{array}{l}\text { Because of adversarial component it cannot guarantee } \\
\text { to capture the dependencies }\end{array}$ \\
\hline
\end{tabular}

\section{DISCUSSION}

In this paper, we reviewed the time delay problem in the teleoperation. In the first section, we reviewed the early approaches and studies in this field. Most of the early methods focused on understanding and estimating the delay. Some work concentrated on the design of systems to reduce the computation load. Next, we focused on reviewing solutions for the time delay problem, which has the potential be used to help mitigate issues with time delay. We surveyed two major areas for time series prediction, which included both statistical and neural network approaches. In Table 1, we compare all the main methods for time series prediction covered here.

The general advantage of the statistical methods is that they do not require training data and tend to be relatively simple methods with clear implementation avenues. Though the statistical methods are the traditional solutions for time series prediction, they cannot model all non-stationary signals. There are some extensions of the ARMA model that can handle non-stationary signals like ARIMA. If the data has seasonality, then other models such as SARIMA/SARIMAX were introduced. However, all these techniques deal with a specific kind of nonstationarity. Another disadvantage of these statistical methods is that they are not suitable for modeling complex tasks. The longer the dependencies, the more difficult to predict. These techniques are more appropriate for short-term predictions. In contrast with statistical models, neural network methods can be used to describe the data without necessarily knowing the distribution of the data. Moreover, by introducing LSTM networks, the literature shows that we can model complicated time series data taking into account many of the past dependencies. It is also possible that neural networks could be used in an adaptive way to change behaviors as more data is available. In this paper, we reviewed new neural network architectures for time series prediction. These methods consist of Seq2Seq and GAN models. The main advantage of these methods over traditional $\mathrm{NN}$ methods is that they are able to take inputs of variable sizes. These methods are promising for time series prediction due to the capability of their architecture to capture the time series distributions.

In the various neural network-based approaches, the relation between input and output is not clearly understood. It is modeled mostly as a black box of weights. In order to have a greater understanding of the mechanism of this transfer function, we point to a new approach by using the concepts of information theory. Information theory allows us to explain better and perhaps control the complex relationships. This idea is beginning to be used in deep learning by Shwartz-Ziv and Tishby (2017) and followed by Alemi et al. (2016) for variational autoencoders.

Readers are directed to the following repository, where several of the promising techniques described here are implemented https://github.com/parinazfa/Recent-Trends-in-TeleoperationTime-DelayMitigation.git.

\section{CONCLUSION}

The time delay problem in teleoperation systems is an important challenge; therefore, several approaches from control-based to deep learning methods have been reviewed in this paper. This survey paper was divided into two parts: first, we covered traditional approaches to mitigating time delay in teleoperation. These methods included stability analysis and predictive methods for teleoperation systems. These methods focused on understanding and estimating the delay. Second, we covered time series prediction methods for mitigating time delay. These methods included the model of user intent or the system using time series prediction techniques. We reviewed statistical and $\mathrm{NN}$ based methods which are applied to mitigating time delay in teleoperation systems. We also reviewed some other deep learning models which may prove to beneficial for mitigation of time delay in teleoperation systems.

We believe that the new machine learning methods for time series prediction open a promising avenue for solving the time delay problem in teleoperation systems. A system that can predict the short-term future may be able to compensate for the time 
delay. It could also be able to adapt to new data and change its mode based on the situation.

Time series prediction opens a new avenue for safety. A related application to teleoperation is based on the prediction capability of time series approaches. If one can predict the immediate future when teleoperating (even in real-time teleoperation), dangerous future events can be mitigated to create safer systems. For example, in telesurgery systems, time series prediction along with real-time surface mapping and registration can warn the surgeon (or even stop or dampen movements) before dangerous tool's movements lead to bleeding, etc. Similarly, in other teleoperation systems, the map of the remote site along with an intelligent overwatch system, can inform the operator about possible dangers and inadvertent movements. Hence, solving the

\section{REFERENCES}

Aburime, W., Schwartz, H., and Givigi, S. (2019). "Compensation for time delays in the navigation of unmanned aerial vehicles," in 2019 IEEE International Systems Conference (SysCon), (Orlando, FL) 1-8.

Ak, R., Fink, O., and Zio, E. (2015). Two machine learning approaches for shortterm wind speed time-series prediction. IEEE Trans. Neural Netw. Learn. Syst. 27, 1734-1747. doi: 10.1109/TNNLS.2015.2418739

Alemi, A. A., Fischer, I., Dillon, J. V., and Murphy, K. (2016). Deep variational information bottleneck. arXiv [Preprint]. arXiv:1612.00410.

Anava, O., Hazan, E., Mannor, S., and Shamir, O. (2013). "Online learning for time series prediction," in Conference on Learning Theory, 172-184.

Anava, O., Hazan, E., and Zeevi, A. (2015). "Online time series prediction with missing data," in International Conference on Machine Learning, 2191-2199.

Anderson, R. J., and Spong, M. W. (1992). “Asymptotic stability for force reflecting teleoperators with time delay," in The International Booktitle of Robotics Research, Vol. 11 (Sage, CA; Thousand Oaks, CA: Sage Publications), 135-149.

Aneiros-Pérez, G., and Vieu, P. (2008). Nonparametric time series prediction: a semi-functional partial linear modeling. J. Multivar. Anal. 99, 834-857. doi: 10.1016/j.jmva.2007.04.010

Arita, R., and Suzuki, S. (2019). "Maneuvering assistance of teleoperation robot based on identification of gaze movement," in 2019 IEEE 17th International Conference on Industrial Informatics (INDIN), (Helsinki) Vol. 1, 565-570.

Bejczy, A. K., and Kim, W. S. (1990). "Predictive displays and shared compliance control for time-delayed telemanipulation," in EEE International Workshop on Intelligent Robots and Systems, Towards a New Frontier of Applications (Ibaraki), 407-412.

Bejczy, A. K., Kim, W. S., and Venema, S. C. (1990). "The phantom robot: predictive displays for teleoperation with time delay," in Proceedings IEEE International Conference on Robotics and Automation, (Cincinnati, $\mathrm{OH}$ ) 546-551.

Belhaj, S., and Tagina, M. (2009). Modeling and prediction of the internet end-to-end delay using recurrent neural networks. J. Netw. 4, 528-535. doi: 10.4304/jnw.4.6.528-535

Benedetti, C., Franchini, M., and Fiorini, P. (2001). "Stable tracking in variable time-delay teleoperation," in Proceedings 2001 IEEE/RSJ International Conference on Intelligent Robots and Systems. Expanding the Societal Role of Robotics in the Next Millennium (Cat. No. 01CH37180), (Maui, HI) Vol. 4, 2252-2257.

Bengio, Y., Simard, P., and Frasconi, P. (1994). Learning long-term dependencies with gradient descent is difficult. IEEE Trans. Neural Netw. 5, 157-166.

Box, G. E., Jenkins, G. M., Reinsel, G. C., and Ljung, G. M. (2015). Time Series Analysis: Forecasting and Control. John Wiley \& Sons.

Box, G. E., and Pierce, D. A. (1970). Distribution of residual autocorrelations in autoregressive-integrated moving average time series models. J. Am. Stat. Assoc. 65, 1509-1526. doi: 10.2307/2284333

Buzan, F. T., and Sheridan, T. B. (1989). "A model-based predictive operator aid for telemanipulators with time delay," in Conference Proceedings, IEEE International Conference on Systems, Man and Cybernetics, (Cambridge, MA), $138-143$. time delay issue with predictive technologies will also have these other important and related applications.

\section{ETHICS STATEMENT}

Written informed consent was obtained from the individual(s) for the publication of any potentially identifiable images or data included in this article.

\section{AUTHOR CONTRIBUTIONS}

HY contributed to the AI sections related to LSTM, GAN, and general editing of the document. All authors contributed to the article and approved the submitted version.

Chen, H., Huang, P., and Liu, Z. (2019). "Modeling and forecasting of time delay about the space robot teleoperation system," in 2019 IEEE 4th International Conference on Advanced Robotics and Mechatronics (ICARM), (Toyonaka) 529-534.

Chen, H., Huang, P., Liu, Z., and Ma, Z. (2020). Time Delay Prediction for Space Telerobot System With a Modified Sparse Multivariate Linear Regression Method, Vol. 166. Elsevier, 330-341. doi: 10.1016/j.actaastro.2019.10.027

Chen, J. Y. (2010). UAV-guided navigation for ground robot tele-operation in a military reconnaissance environment. Ergonomics 53, 940-950. doi: 10.1080/00140139.2010.500404

Chintamani, K., Cao, A., Ellis, R. D., and Pandya, A. K. (2009). Improved telemanipulator navigation during display-control misalignments using augmented reality cues. IEEE Trans. Syst. Man Cybern. 40, 29-39. doi: 10.1109/TSMCA.2009.2030166

Cho, K., Van Merriënboer, B., Bahdanau, D., and Bengio, Y. (2014). On the properties of neural machine translation: encoder-decoder approaches. arXiv:1409.1259.

Choi, B. (2012). ARMA Model Identification. Springer Science \& Business Media.

Colgate, J. E. (1991). "Power and impedance scaling in bilateral manipulation," in Proceedings. 1991 IEEE International Conference on Robotics and Automation, (Los Alamitos, CA) 2292-2297.

Connor, J. T., Martin, R. D., and Atlas, L. E. (1994). Recurrent neural networks and robust time series prediction. IEEE Trans. Neural Netw. 5, 240-254.

Desoer, C. A., and Vidyasagar, M. (1975). Feedback Systems: Input-Output Properties, Vol. 55. SIAM.

Donahue, J., Krähenbühl, P., and Darrell, T. (2016). Adversarial feature learning. arXiv [preprint] arXiv:1605.09782.

Du, S., Li, T., and Horng, S.-J. (2018). "Time series forecasting using sequenceto-sequence deep learning framework," in 2018 9th International Symposium on Parallel Architectures, Algorithms and Programming (PAAP), (Taipei) $171-176$.

Eslamian, S., Reisner, L. A., and Pandya, A. K. (2020). Development and evaluation of an autonomous camera control algorithm on the da Vinci surgical system. Int. J. Med. Robot. Comput. Assist. Surg. 16:e2036. doi: 10.1002/rcs.2036

Fang, B., Ma, X., Wang, J., and Sun, F. (2020). Vision-based posture-consistent teleoperation of robotic arm using multi-stage deep neural network. Robot. Auton. Syst. 131:103592. doi: 10.1016/j.robot.2020.103592

Fang, B., Sun, F., Liu, H., Guo, D., Chen, W., and Yao, G. (2017). Robotic teleoperation systems using a wearable multimodal fusion device. Int. J. Adv. Robot. Syst. 14:1729881417717057. doi: 10.1177/1729881417717057

Faruk, D. Ö. (2010). A hybrid neural network and arima model for water quality time series prediction. Eng. Appl. Artif. Intell. 23, 586-594 doi: 10.1016/j.engappai.2009.09.015

Ferrell, W. R. (1965). Remote manipulation with transmission delay. IEEE Trans. Hum. Factors Electron. HFE-6, 24-32.

Ferrell, W. R., and Sheridan, T. B. (1967). Supervisory control of remote manipulation. IEEE Spectr. 4, 81-88.

Fong, C., Dotson, R., and Bejczy, A. (1986). "Distributed microcomputer control system for advanced teleoperation," in 1986 IEEE International Conference on Robotics and Automation, Vol. 3, (San Francisco, CA) 987-995. 
Ganjefar, S., Momeni, H., and Janabi-Sharifi, F. (2002). “Teleoperation systems design using augmented wave-variables and smith predictor method for reducing time-delay effects," in Proceedings of the IEEE Internatinal Symposium on Intelligent Control, (Vancouver, BC) 333-338.

Giles, C. L., Lawrence, S., and Tsoi, A. C. (2001). Noisy time series prediction using recurrent neural networks and grammatical inference. Mach. Learn. 44, 161-183. doi: 10.1023/A:1010884214864

Goertz, R. C., and Thompson, W. M. (1954). "Electronically controlled manipulator," in Nucleonics (US) Ceased Publication, Vol. 12 (Lemont, IL: Argonne National Lab), 46-47.

Goldberg, K., Mascha, M., Gentner, S., Rothenberg, N., Sutter, C., and Wiegley, J. (1995). "Desktop teleoperation via the world wide web," in Proceedings of 1995 IEEE International Conference on Robotics and Automation, Vol. 1, (Nagoya) 654-659.

Goodfellow, I., Pouget-Abadie, J., Mirza, M., Xu, B., Warde-Farley, D., Ozair, S., et al. (2014). "Generative adversarial nets," in Advances in Neural Information Processing Systems, (Montréal, QC) 2672-2680.

Hannaford, B. (1989). A design framework for teleoperators with kinesthetic feedback. IEEE Trans. Robot. Autom. 5, 426-434.

Hirzinger, G., Heindl, J., and Landzettel, K. (1989). "Predictive and knowledgebased telerobotic control concepts," in Proceedings, 1989 International Conference on Robotics and Automation, (Scottsdale, AZ) 1768-1777.

Ho, J., and Ermon, S. (2016). "Generative adversarial imitation learning," in Advances in Neural Information Processing Systems, (Barcelona) 4565-4573.

Hochreiter, S., and Schmidhuber, J. (1997). Long short-term memory. Neural Comput. 9, 1735-1780.

Hokayem, P. F., and Spong, M. W. (2006). Bilateral teleoperation: an historical survey. Automatica 42, 2035-2057. doi: 10.1016/j.automatica.2006.06.027

Hu, T., Huang, X., and Tan, Q. (2012). "Time delay prediction for space teleoperation based on non-gaussian auto-regressive model," in 2012 Proceedings of International Conference on Modelling, Identification and Control, (Wuhan, Hubei) 567-572.

Hua, J., Cui, Y., Yang, Y., and Li, H. (2013). "Analysis and prediction of jitter of internet one-way time-delay for teleoperation systems," in 2013 11th IEEE International Conference on Industrial Informatics (INDIN), (Bochum) 612-617.

Hyndman, R. J., and Athanasopoulos, G. (2018). Forecasting: Principles and Practice. OTexts.

Jung, Y., Han, K., and Bae, J. (2019). A tele-operated display with a predictive display algorithm. IEEE Access 7, 154447-154456. doi: 10.1109/ACCESS.2019.2948879

Kalekar, P. S. (2004). Time series forecasting using holt-winters exponential smoothing.

Karim, F., Majumdar, S., Darabi, H., and Chen, S. (2017). LSTM fully convolutional networks for time series classification. Mach. Learn. 6, 1662-1669. doi: 10.1109/ACCESS.2017.2779939

Kebria, P. M., Abdi, H., Dalvand, M. M., Khosravi, A., and Nahavandi, S. (2018). Control methods for internet-based teleoperation systems: a review. IEEE Trans. Hum. Mach. Syst. 49, 32-46. doi: 10.1109/THMS.2018.2878815

Kim, P., MacLennan, C. J., and Tang, Z. (2010). Modeling Sequence and Time Series Data in Predictive Analytics. US Patent 7,747,641.

Koochali, A., Schichtel, P., Dengel, A., and Ahmed, S. (2019). Probabilistic forecasting of sensory data with generative adversarial networks-forGAN. IEEE Access 7, 63868-63880. doi: 10.1109/ACCESS.2019.2915544

Li, Z., and Su, C.-Y. (2013). Neural-adaptive control of single-master-multipleslaves teleoperation for coordinated multiple mobile manipulators with timevarying communication delays and input uncertainties. IEEE Trans. Neural Netw. Learn. Syst. 24, 1400-1413. doi: 10.1109/TNNLS.2013.2258681

Lipton, Z. C., Berkowitz, J., and Elkan, C. (2015). A critical review of recurrent neural networks for sequence learning. arXiv [Preprint]. arXiv:1506.00019.

Livatino, S., De Paolis, L. T., D’Agostino, M., Zocco, A., Agrimi, A., De Santis, A., et al. (2014). Stereoscopic visualization and 3-d technologies in medical endoscopic teleoperation. IEEE Trans. Indust. Electron. 62, 525-535. doi: 10.1109/TIE.2014.2334675

Lorek, K. S., and Willinger, G. L. (1996). A multivariate time-series prediction model for cash-flow data. Account. Rev. 71, 81-102.

Lotter, W., Kreiman, G., and Cox, D. (2016). Deep predictive coding networks for video prediction and unsupervised learning. arXiv [Preprint]. arXiv:1605.08104.
Lu, Z., Huang, P., and Liu, Z. (2017). Predictive approach for sensorless bimanual teleoperation under random time delays with adaptive fuzzy control. IEEE Trans. Indus. Electron. 65, 2439-2448. doi: 10.1109/TIE.2017.2745445

Lucas, N. P., Pandya, A. K., and Ellis, R. D. (2012). "Review of multi-robot taxonomy, trends, and applications for defense and space," in Unmanned Systems Technology XIV, Vol. 8387 (Baltimore, MD: International Society for Optics and Photonics), 83871N.

Lum, M. J., Rosen, J., King, H., Friedman, D. C., Lendvay, T. S., Wright, A. S., et al. (2009). "Teleoperation in surgical robotics-network latency effects on surgical performance," in 2009 Annual International Conference of the IEEE Engineering in Medicine and Biology Society, (Minneapolis, MN) 6860-6863.

Madder, R. D., VanOosterhout, S., Mulder, A., Bush, J., Martin, S., Rash, A. J., et al. (2019). "Network latency and long-distance robotic telestenting: exploring the potential impact of network delays on telestenting performance," in Catheterization and Cardiovascular Interventions (Wiley Online Library).

Madni, A., Chu, Y.-y., and Freedy, A. (1983). "Intelligent interface for remote supervision and control of underwater manipulation," in Proceedings OCEANS'83, (San Francisco, CA) 106-110.

Mariet, Z., and Kuznetsov, V. (2019). "Foundations of sequence-to-sequence modeling for time series," in The 22nd International Conference on Artificial Intelligence and Statistics, (Naha, Okinawa) 408-417.

Mikolov, T., Karafiát, M., Burget, L., Černockỳ, J., and Khudanpur, S. (2010). "Recurrent neural network based language model," in Eleventh Annual Conference of the International Speech Communication Association. (Makuhari, Chiba).

Mills, T. C., and Mills, T. C. (1991). Time Series Techniques for Economists. Cambridge University Press.

Mirfakhrai, T., and Payandeh, S. (2002). "A delay prediction approach for teleoperation over the internet," in Proceedings 2002 IEEE International Conference on Robotics and Automation (Cat. No. 02CH37292), (Washington, DC) Vol. 2, 2178-2183.

Mishra, A. K., and Desai, V. R. (2005). Drought forecasting using stochastic models. Stoch Environ Res Ris Assess 19, 326-339. doi: 10.1007/s00477-005-0238-4

Moayedi, H. Z., and Masnadi-Shirazi, M. (2008). "Arima model for network traffic prediction and anomaly detection," in 2008 International Symposium on Information Technology, Vol. 4, (Kuala Lumpur) 1-6.

Mondal, P., Shit, L., and Goswami, S. (2014). "Study of effectiveness of time series modeling (arima) in forecasting stock prices," in International Booktitle of Computer Science, Engineering and Applications, Vol. 4 (Academy \& Industry Research Collaboration Center (AIRCC)), 13.

Montgomery, D. C., Jennings, C. L., and Kulahci, M. (2015). Introduction to Time Series Analysis and Forecasting. John Wiley \& Sons.

Munir, S., and Book, W. J. (2002). Internet-based teleoperation using wave variables with prediction. IEEE/ASME Trans. Mechatron. 7, 124-133. doi: 10.1109/TMECH.2002.1011249

Muradore, R., and Fiorini, P. (2016). A review of bilateral teleoperation algorithms. Acta Polytech. Hungar. 13, 191-208. doi: 10.12700/APH.13.1.2016.1.13

Niemeyer, G., and Slotine, J.-J. (1991a). Stable adaptive teleoperation. IEEE J. Ocean. Eng. 16, 152-162.

Niemeyer, G., and Slotine, J.-J. (1991b). "Transient shaping in force-reflecting teleoperation," in Fifth International Conference on Advanced Robotics' Robots in Unstructured Environments, (Pisa) 261-266.

Nuño, E., Basa nez, L., and Ortega, R. (2011). Passivity-based control for bilateral teleoperation: a tutorial. Automatica 47, 485-495. doi: 10.1016/j.automatica.2011.01.004

Oboe, R. (2003). "Force-reflecting teleoperation over the internet: the JBIT project," in Proceedings of the IEEE, Vol. 91, 449-462.

Orosco, R. K., Lurie, B., Matsusaki, T., Funk, E. K., Divi, V., Holsinger, F. C., et al. (2020). Compensatory motion scaling for time-delayed robotic surgery. Surg. Endosc. doi: 10.1007/s00464-020-07681-7

Park, D., Hoshi, Y., and Kemp, C. C. (2018). A multimodal anomaly detector for robot-assisted feeding using an lstm-based variational autoencoder. IEEE Robot. Autom. Lett. 3, 1544-1551. doi: 10.1109/LRA.2018.28 01475

Parmezan, A. R. S., Souza, V. M., and Batista, G. E. (2019). Evaluation of statistical and machine learning models for time series prediction: identifying the stateof-the-art and the best conditions for the use of each model. Inform. Sci. 484, 302-337. doi: 10.1016/j.ins.2019.01.076 
Polat, I., and Scherer, C. W. (2012). Stability analysis for bilateral teleoperation: an IQC formulation. IEEE Trans. Robot. 28, 1294-1308. doi: 10.1109/TRO.2012.2209230

Poskitt, D. S. (2016). Vector autoregressive moving average identification for macroeconomic modeling: a new methodology. J. Econ. 192, 468-484. doi: 10.1016/j.jeconom.2016.02.011

Radford, A., Metz, L., and Chintala, S. (2015). Unsupervised representation learning with deep convolutional generative adversarial networks. arXiv:1511.06434

Raju, G. J., Verghese, G. C., and Sheridan, T. B. (1989). "Design issues in 2-port network models of bilateral remote manipulation," in Proceedings, 1989 International Conference on Robotics and Automation, (Scottsdale, AZ) 1316-1321.

Rojas, I., Valenzuela, O., Rojas, F., Guillén, A., Herrera, L. J., Pomares, H., et al. (2008). Soft-computing techniques and ARMA model for time series prediction. Neurocomputing 71, 519-537. doi: 10.1016/j.neucom.2007.07.018

Rosen, J., and Hannaford, B. (2006). Doc at a distance. IEEE Spectr. 43, 34-39. doi: 10.1109/MSPEC.2006.1705774

Salinas, D., Flunkert, V., Gasthaus, J., and Januschowski, T. (2020). DeepAR: Probabilistic forecasting with autoregressive recurrent networks. Int. J. Forecast. 36, 1181-1191. doi: 10.1016/j.ijforecast.2019.07.001

Saltaren, R., Aracil, R., Alvarez, C., Yime, E., and Sabater, J. M. (2007). Field and service applications-exploring deep sea by teleoperated robot-an underwater parallel robot with high navigation capabilities. IEEE Robot. Autom. Mag. 14, 65-75. doi: 10.1109/MRA.2007.905502

Sanchez, L. A., Le, M., Liu, C., Zemiti, N., and Poignet, P. (2012). “The impact of interaction model on stability and transparency in bilateral teleoperation for medical applications," in 2012 IEEE International Conference on Robotics and Automation, (Saint Paul, MN) 1607-1613.

Sato, T., and Hirai, S. (1987). Language-aided robotic teleoperation system (larts) for advanced teleoperation. IEEE J. Robot. Autom. 3, 476-481.

Shen, S., Song, A., Li, T., and Li, H. (2019). Time Delay Compensation for Nonlinear Bilateral Teleoperation: A Motion Prediction Approach, Vol. 41. Sage; London: SAGE Publications, 4488-4498.

Sheridan, T. B. (1992). Telerobotics, Automation, and Human Supervisory Control. MIT Press.

Shmueli, G. (2010). To explain or to predict? Stat. Sci. 25, 289-310. doi: $10.1214 / 10$-STS330

Shwartz-Ziv, R., and Tishby, N. (2017). Opening the black box of deep neural networks via information. arXiv [Preprint]. arXiv:1703.00810.

Sirouspour, S. (2005). Modeling and control of cooperative teleoperation systems. IEEE Trans. Robot. 21, 1220-1225. doi: 10.1109/TRO.2005.852254

Sirouspour, S., and Shahdi, A. (2006). Model predictive control for transparent teleoperation under communication time delay. IEEE Trans. Robot. 22, 1131-1145. doi: 10.1109/TRO.2006.882939

Stark, L., Kim, W.-S., Tendick, F., Hannaford, B., Ellis, S., Denome, M., et al. (1987). Telerobotics: display, control, and communication problems. IEEE J. Robot. Autom. 3, 67-75.

Stramigioli, S., Van Der Schaft, A., Maschke, B., and Melchiorri, C. (2002). Geometric scattering in robotic telemanipulation. IEEE Trans. Robot. Autom. 18, 588-596. doi: 10.1109/TRA.2002.802200

Su, H., Hu, Y., Karimi, H. R., Knoll, A., Ferrigno, G., and De Momi, E. (2020a). Improved recurrent neural network-based manipulator control with remote center of motion constraints: experimental results. Neural Netw. 131, 291-299. doi: 10.1016/j.neunet.2020.07.033

Su, H., Qi, W., Yang, C., Sandoval, J., Ferrigno, G., and De Momi, E. (2020b). Deep neural network approach in robot tool dynamics identification for bilateral teleoperation. IEEE Robot. Autom. Lett. 5, 2943-2949. doi: 10.1109/LRA.2020.2974445

Sun, D., Naghdy, F., and Du, H. (2014). Application of wave-variable control to bilateral teleoperation systems: a survey. Annu. Rev. Control 38, 12-31. doi: 10.1016/j.arcontrol.2014.03.002

Sutskever, I., Vinyals, O., and Le, Q. V. (2014). "Sequence to sequence learning with neural networks," in Advances in Neural Information Processing Systems, (Montréal, QC) 3104-3112.

Tugal, H., Carrasco, J., Falcon, P., and Barreiro, A. (2016). Stability analysis of bilateral teleoperation with bounded and monotone environments via zames-falb multipliers. IEEE Trans. Control Syst. Technol. 25, 1331-1344. doi: 10.1109/TCST.2016.2601289

Uddin, R., and Ryu, J. (2016). Predictive control approaches for bilateral teleoperation. Annu. Rev. Control 42, 82-99. doi: 10.1016/j.arcontrol.2016.09.003

Varkonyi, T. A., Rudas, I. J., Pausits, P., and Haidegger, T. (2014). "Survey on the control of time delay teleoperation systems," in IEEE 18th International Conference on Intelligent Engineering Systems INES 2014, (Tihany) 89-94.

Venugopalan, S., Rohrbach, M., Donahue, J., Mooney, R., Darrell, T., and Saenko, K. (2015). "Sequence to sequence-video to text," in Proceedings of the IEEE International Conference on Computer Vision, (Santiago) 4534-4542.

Wasserman, L. (2013). All of Statistics: A Concise Course in Statistical Inference. Springer Science \& Business Media.

Whitney, D. (1969). State space models of remote manipulation tasks. IEEE Trans. Autom. Control 14, 617-623.

Xi, N., and Tarn, T. J. (2000). Stability analysis of non-time referenced internet-based telerobotic systems. Robot. Auton. Syst. 32, 173-178. doi: 10.1016/S0921-8890(99)00118-9

Xingjian, S., Chen, Z., Wang, H., Yeung, D.-Y., Wong, W.-K., and Woo, W.-c. (2015). "Convolutional Lstm network: a machine learning approach for precipitation nowcasting," in Advances in Neural Information Processing Systems, (Montréal, QC) 802-810.

Yang, C., Guo, Z., and Xian, L. (2019). "Time series data prediction based on sequence to sequence model," in IOP Conference Series: Materials Science and Engineering, Vol. 692 (Wuhan: IOP Publishing), 012047.

Yang, C., Luo, J., Liu, C., Li, M., and Dai, S.-L. (2018). Haptics electromyography perception and learning enhanced intelligence for teleoperated robot. IEEE Trans. Autom. Sci. Eng. 16, 1512-1521. doi: 10.1109/TASE.2018. 2874454

Yang, G.-Z., Nelson, B. J., Murphy, R. R., Choset, H., Christensen, H., Collins, S. H., et al. (2020). Combating COVID-19-the role of robotics in managing public health and infectious diseases. Sci. Robot. 5:eabb5589. doi: 10.1126/scirobotics.abb5589

Yang, Q., Piantanida, P., and Gündüz, D. (2017). The multi-layer information bottleneck problem. arXiv [Preprint]. arXiv:1711.05102.

Ye, X., Meng, M.-H., Liu, P. X., and Li, G. (2002). "Statistical analysis and prediction of round trip delay for internet-based teleoperation," in IEEE/RSJ International Conference on Intelligent Robots and Systems, Vol. 3, (Lausanne) 2999-3004.

Yoon, J., Jarrett, D., and van der Schaar, M. (2019). "Time-series generative adversarial networks," in Advances in Neural Information Processing Systems, (Vancouver, BC) 5509-5519.

Zec, E. L., Arnelid, H., and Mohammadiha, N. (2019). "Recurrent conditional gans for time series sensor modeling," in Time Series Workshop at International Conference on Machine Learning (Long Beach, CA).

Zhang, K., Zhong, G., Dong, J., Wang, S., and Wang, Y. (2019). Stock market prediction based on generative adversarial network. Proc. Comput. Sci. 147, 400-406. doi: 10.1016/j.procs.2019.01.256

Zheng, Y., Brudnak, M. J., Jayakumar, P., Stein, J. L., and Ersal, T. (2019). A delay compensation framework for predicting heading in teleoperated ground vehicles. IEEE/ASME Trans. Mechatron. 24, 2365-2376. doi: 10.1109/TMECH.2019.2936313

Zhu, L., and Laptev, N. (2017). "Deep and confident prediction for time series at Uber," in 2017 IEEE International Conference on Data Mining Workshops (ICDMW), (New Orleans, LA) 103-110.

Conflict of Interest: The authors declare that the research was conducted in the absence of any commercial or financial relationships that could be construed as a potential conflict of interest.

Copyright (c) 2020 Farajiparvar, Ying and Pandya. This is an open-access article distributed under the terms of the Creative Commons Attribution License (CC BY). The use, distribution or reproduction in other forums is permitted, provided the original author(s) and the copyright owner(s) are credited and that the original publication in this journal is cited, in accordance with accepted academic practice. No use, distribution or reproduction is permitted which does not comply with these terms. 